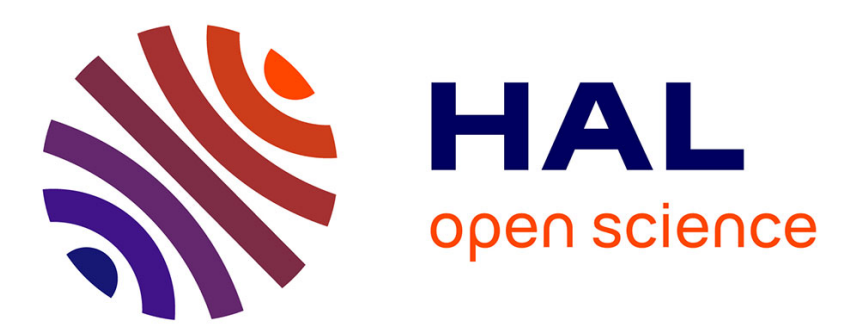

\title{
Absolute stability and the Lagrange-Dirichlet theorem with monotone multivalued mappings
}

Bernard Brogliato

\section{To cite this version:}

Bernard Brogliato. Absolute stability and the Lagrange-Dirichlet theorem with monotone multivalued mappings. RR-4794, INRIA. 2003. inria-00071792

\section{HAL Id: inria-00071792 \\ https://hal.inria.fr/inria-00071792}

Submitted on 23 May 2006

HAL is a multi-disciplinary open access archive for the deposit and dissemination of scientific research documents, whether they are published or not. The documents may come from teaching and research institutions in France or abroad, or from public or private research centers.
L'archive ouverte pluridisciplinaire $\mathbf{H A L}$, est destinée au dépôt et à la diffusion de documents scientifiques de niveau recherche, publiés ou non, émanant des établissements d'enseignement et de recherche français ou étrangers, des laboratoires publics ou privés. 
INSTITUT NATIONAL DE RECHERCHE EN INFORMATIQUE ET EN AUTOMATIQUE

\section{Absolute stability and the Lagrange-Dirichlet theorem with monotone multivalued mappings}

Bernard Brogliato

$\mathbf{N}^{\circ} 4794$

April 2003

THÈME 4 



\title{
Absolute stability and the Lagrange-Dirichlet theorem with monotone multivalued mappings
}

\author{
Bernard Brogliato * \\ Thème 4 - Simulation et optimisation \\ de systèmes complexes \\ Projet Bipop \\ Rapport de recherche $n^{\circ} 4794$ - April 2003 - 20 pages
}

\begin{abstract}
This note presents an extension of the absolute stability problem and of the Lagrange-Dirichlet theorem, when the nonlinearities entering the model are considered within the class of monotone multivalued mappings (consequently including operators with piecewise-linear graphs that may represent physical effects like Coulomb friction, dead-zones, saturations, elasto-plasticity, and unilateral constraints).
\end{abstract}

Key-words: Absolute stability, nonsmooth Lyapunov functions, multivalued monotone mappings, Moreau's sweeping process, passivity, convex analysis.

* INRIA Rhône-Alpes, ZIRST Montbonnot, 655 avenue de l'Europe, 38334 Saint-Ismier, France, Bernard.Brogliato@inrialpes.fr 


\section{Stabilité absolue et le théorème de Lagrange-Dirichlet avec des opérateurs monotones multivalués}

Résumé : Cette note concerne l'extension du problème de la stabilité absolue et du théorème de Lagrange-Dirichlet, lorsque l'on considère des opérateurs monotones multivalués dans la boucle de retour.

Mots-clés : Stabilité absolue, fonction de Lyapunov non-lisse, opérateurs monotones multivalués, processus de rafle de Moreau,passivité, analyse convexe. 


\section{Introduction}

The absolute stability problem (and its various forms known as the passivity theorems) and the Lagrange-Dirichlet theorem are both fundamental tools for the study of the stability of nonlinear dynamical systems [1] [2] [21]. The first one concerns the study of the stability of a linear Positive Real transfer function $G(s)$ (or more generally of any dissipative system) with a nonlinear static feedback characteristic, see figure 1 . The second one gives a criterion for the stability of Lagrangian mechanical systems. In this note we first extend theorem 5.6.18 in [1] $\left({ }^{1}\right)$ to the case when the static nonlinearities are multivalued monotone mappings (thus in particular allowing the nonlinearity to belong to the sector $[0,+\infty]$ and not restricted to the sector $[0,+\infty)$ ). Then we extend the Lagrange-Dirichlet theorem to the case of mechanical Lagrangian systems subject to unilateral [4] (or inequality) frictionless constraints. We make use of the convex analysis framework as developed by Rockafellar and Moreau [6] [7]. Before stating the two main results, we briefly recall the conditions which guarantee that a given state $x_{0}$ is a fixed point of the dynamical system considered. They take the form of generalized equations $\left({ }^{2}\right)$. The interest for extending the above stability tools towards the case when the feedback nonlinearity is a multivalued monotone mapping, lies in the fact that such operators are widely used in physical modelling (e.g. in mechanics [19] [20] [24] [25]). Though monotonicity clearly implies dissipativity of the said operator, rendering such extensions more or less intuitively clear, the analysis has not, to the best of our knowledge, yet been done.

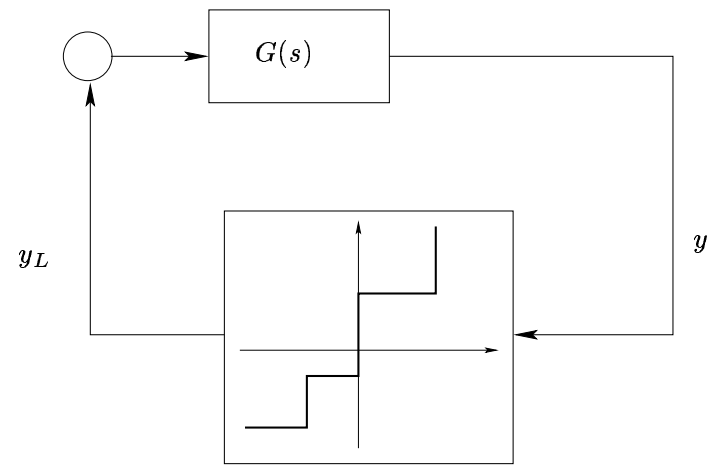

Figure 1: Absolute stability with monotone multivalued mappings.

The note is organized as follows: in section 2 we recall some basic concepts of convex analysis. Section 3 is devoted to the absolute stability problem, and section 4 contains the

\footnotetext{
${ }^{1} G(s)$ in figure 1 is a Strictly Positive Real transfer matrix and the operator $P L: y \mapsto y_{L}$ satisfies $P L(0)=0, y^{T} P L(y) \geq 0 \forall t \geq 0$ and $\forall y \in \mathbb{R}^{m}$, i.e. PL belongs to the sector $[0,+\infty)$.

${ }^{2}$ i.e. certain types of relations that are similar to equations except that one side of the relation is multivalued.
}

$\mathrm{RR} \mathrm{n}^{\circ} 4794$ 
material concerning the Lagrange-Dirichlet theorem applied to Lagrangian systems subject to unilateral constraints on the position. Conclusions end the note in section 5 .

\section{Basic facts in convex and nonsmooth analysis}

All the material in this section is taken from [6] [7] and only aims at introducing the tools which are needed in this note.

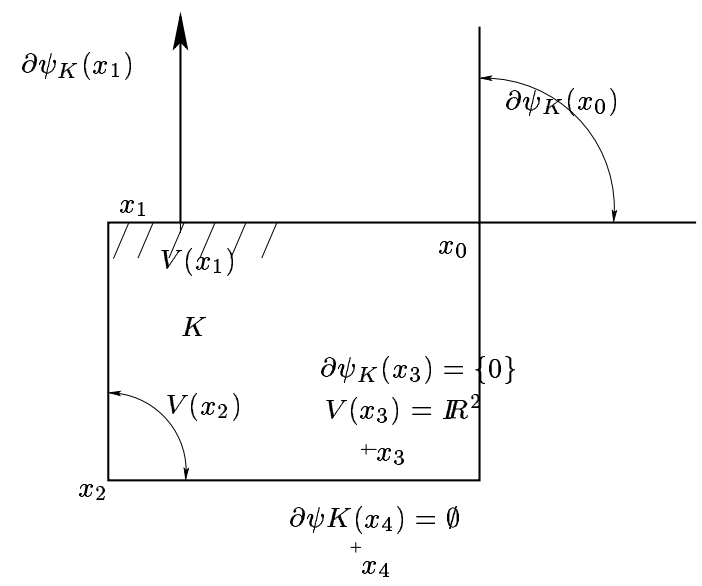

Figure 2: Tangent and normal cones.

Let $K \subset \mathbb{R}^{n}$ denote a convex set. Its indicator function is defined as

$$
\psi_{K}(x)= \begin{cases}0 & \text { if } x \in K \\ +\infty & \text { if } x \notin K\end{cases}
$$

A convex function $f(\cdot)$ satisfies $f(\lambda x+(1-\lambda) y) \leq \lambda f(x)+(1-\lambda) f(y)$ for all $0<\lambda<1$, and for all $x$ and $y$ in its (convex) domain of definition. The subdifferential of a convex function $f(\cdot)$ at $y$ is denoted as $\partial f(y)$ and is the set of subgradients, i.e. vectors $\gamma$ satisfying $f(x)-f(y) \geq \gamma^{T}(x-y)$ for all $x$. The subdifferential of the indicator of $K$ (which is convex if $K$ is convex) is given by

$$
\partial \psi_{K}(x)= \begin{cases}\{0\} & \text { if } x \in \operatorname{Int}(K) \\ N_{K}(x) & \text { if } x \in \partial K \\ \emptyset & \text { if } x \notin K\end{cases}
$$

INRIA 
where $\partial K$ is the boundary of $K$, and $N_{K}(x)=\left\{z \mid z^{T}(\zeta-x) \leq 0, \forall \zeta \in K\right\}$ is the outwards normal cone to $K$ at $x$. Notice that $0 \in N_{K}(x)$ and that we have drawn the sets $x+N_{K}(x)$ rather than $N_{K}(x)$. The set in (2) is the subdifferential from convex analysis. However when $K$ is not convex it is possible to extend (2) using a different definition of the subdifferential, see [15, proposition 2.4.12]. This will be useful when we deal with Lyapunov stability of mechanical systems with non-convex admissible configuration subspaces in section 4 .

Remark 1 The symbol $\partial$ is used in three different meanings in this paper: boundary of a set, subdifferential and partial derivative. Since these notations are classical we choose not to change them.

The inwards tangent cone $V(x)$ is the polar cone to $N_{K}(x)$ and is defined as $V(x)=$ $\left\{z \mid \forall \zeta \in N_{K}(x), \zeta^{T} z \leq 0\right\}$. It is also sometimes denoted as $T_{K}(x)$ in the literature. Both the normal and the tangent cones are convex sets. If the set $K$ is defined as $\{x \mid h(x) \geq 0\}$ for some differentiable function $h: \mathbb{R}^{n} \rightarrow \mathbb{R}^{m}$, then an alternative definition of the tangent cone at $x$ is [8]

$$
V(x)=\left\{v \in \mathbb{R}^{n} \mid v^{T} \nabla h_{i}(x) \geq 0, \forall i \in J(x)\right\}
$$

with $J(x)=\left\{i \in\{1, \ldots, m\} \mid h_{i}(x) \leq 0\right\}$. One notes that this definition coincides with the first one as long as $x \in K$, and that $K$ needs not be convex to define $V(x)$ in (3). We shall use some material from [8] in section 4 . Some examples are depicted in figure 2, see also [4].

A mapping $\rho(\cdot)$ from $X$ to $Y$ is said multivalued if it assigns to each element $x$ of $X$ a subset $\rho(x)$ of $Y$ (which may be empty, contain just one element, or contain several elements). The mappings whose graphs are in figure 3 (c) (d) (e) (f) are multivalued [19]. A multivalued mapping $\rho(\cdot)$ is monotone if $\left(x-x^{\prime}\right)^{T}\left(y-y^{\prime}\right) \geq 0$ for any couples $(x, y)$ and $\left(x^{\prime}, y^{\prime}\right)$ in its graph. When $n=1$ monotone mappings correspond to completely non-decreasing curves. Let $D(\rho)=\{x \mid x \in X, \rho(x) \neq \emptyset\}$ be the domain of $\rho(\cdot)$. A monotone mapping $\rho(\cdot)$ is maximal if for any $x \in X$ and any $y \in Y$ such that $\left\langle y-y_{1}, x-x_{1}\right\rangle \geq 0$ for any $x_{1} \in D(\rho)$ and any $y_{1} \in \rho\left(x_{1}\right)$, then $y \in \rho(x)$. Complete nondecreasing curves in $\mathbb{R}^{2}$ are the graphs of maximal monotone mappings. Another interpretation is that the graph of a maximal monotone mapping cannot be enlarged without destroying the monotonicity (hence the notion of maximality). The subdifferential $\partial \varphi(\cdot)$ of a convex lower semicontinuous function on $\mathbb{R}^{n}$ is a monotone mapping. Examples of monotone mappings $(n=1)$ are depicted in figure 3. They may represent various physical laws, like dead-zone (a), saturation or elasto-plasticity (b), corner law - unilateral effects, ideal diode characteristic - (c), Coulomb friction (d), MOS transistor ideal charateristic (e), unilateral and adhesive effects (f) [20]. One has for instance $\varphi(x)=\psi_{\mathbb{R}^{-}}(x)$ in figure $3(\mathrm{c}), \varphi(x)=|x|+\frac{x^{2}}{2}$ for (d), $\varphi(x)=$ $\psi_{(-\infty, a]}(x)-\psi_{[-a,+\infty)}(x)+\left\{\begin{array}{l}\frac{a-b}{2}(x-b)^{2} \text { if }|x| \geq b \\ 0 \text { if }|x|<b\end{array}\right.$ for (e). If $\varphi\left(x_{1}, \cdots, x_{m}\right)=\mu_{1}\left|x_{1}\right|+$ $\cdots+\mu_{m}\left|x_{m}\right|+\frac{1}{2} x^{T} x$, then $\partial \varphi(0)=\left(\left[-\mu_{1}, \mu_{1}\right], \cdots,\left[-\mu_{m}, \mu_{m}\right]\right)^{T}$. 


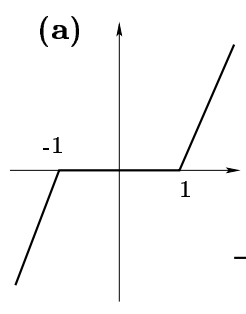

(d)

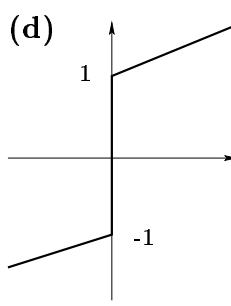

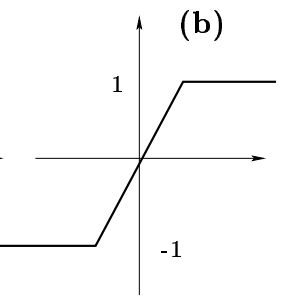

(e)

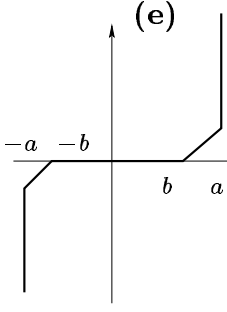

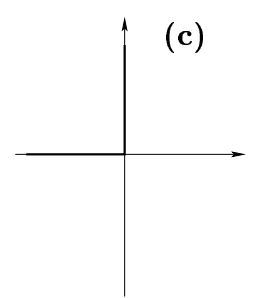

(f)

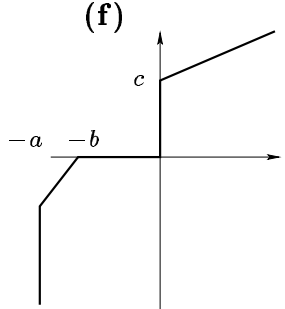

Figure 3: Monotone mappings (one-dimensional case).

\section{The absolute stability problem}

The absolute stability problem consists of studying the stability of a system as in figure 1 where $G(s)$ is a positive real transfer function and the feedback branch contains a sector static nonlinearity. It is of interest to extend this result to the case where the operator $P L: y \in \mathbb{R}^{l} \mapsto y_{L} \in \mathbb{R}^{l}$ is a monotone operator.

The state space equations of the system in figure 1 are given by

$$
\left\{\begin{array}{l}
\dot{x} \stackrel{\text { a.e. }}{=} A x-B y_{L} \\
y=C x \\
y_{L} \in \partial \varphi(y)
\end{array}\right.
$$

where $y, y_{L} \in \mathbb{R}^{m}, x \in \mathbb{R}^{n}$ and a.e. means almost everywhere in the Lebesgue measure. The fixed points of $(4)$ can be characterized with the generalized equation $0 \in\left\{A x_{0}\right\}-$ $B \partial \varphi\left(C x_{0}\right)$. Let us assume that

a) $G(s)=C(s I-A)^{-1} B$, with $(A, B, C)$ a minimal representation, is a Strictly Positive Real (SPR) transfer matrix. In particular this implies that there exists positive definite matrices $P=P^{T}$ and $Q=Q^{T}$ such that $P A+A^{T} P=-Q$ and $B^{T} P=C$ [2].

b) $\varphi: \mathbb{R}^{m} \rightarrow \mathbb{R} \cup\{+\infty\}$ is convex lower semicontinuous, so that $\partial \varphi$ is a maximal monotone multivalued mapping (see e.g. [16, example 2.3.4]). 
Lemma 1 Let assumptions a) and b) hold. If $C x(0) \in \operatorname{dom} \partial \varphi$, then the system in (4) has a unique absolutely continuous $(\mathrm{AC})$ solution on $[0,+\infty)$.

Proof: Let $R$ be the square root of $P$, i.e. $R=R^{T}>0, R R=P$. Consider the convex lower semicontinuous function $f: \mathbb{R}^{n} \rightarrow \mathbb{R} \cup\{+\infty\}$ defined by $f(z)=\varphi\left(C R^{-1} z\right)$. Using a) shows that $\operatorname{Ker}\left(C^{T}\right)=\{0\}$ so that $\operatorname{Im}\left(C R^{-1}\right)=\operatorname{Im}(C)=\mathbb{R}^{m}$. From [6, theorem 2.3.9] it follows that $\partial f(z)=R^{-1} C^{T} \partial \varphi\left(C R^{-1} z\right)$. Let us prove that the system

$$
\left\{\begin{array}{l}
\dot{z} \in R A R^{-1} z-\partial f(z) \\
z(0)=R x(0)
\end{array}\right.
$$

has a unique $\mathrm{AC}$ solution on $[0,+\infty)$. First, to say that $C x(0) \in \operatorname{dom} \partial \varphi$ is to say that $C R^{-1} z(0) \in \operatorname{dom} \partial \varphi$, and this just means that $z(0) \in \operatorname{dom} \partial f$. Second, it follows from the Kalman-Yakubovich-Popov lemma that $R A R^{-1}+\left(R A R^{-1}\right)^{T}$ is negative definite [2, §3.3.1]. Therefore the multivalued mapping $-R A R^{-1}+\partial f$ is maximal monotone [16, lemma 2.4]. Consequently the existence and uniqueness result follows from [16, theorem 3.1].

Now set $x(t)=R^{-1} z(t)$. It is straightforward to check that $x(t)$ is a solution of the system in (4). Actually the system in (5) is deduced from (4) by the change of state vector $z=R x$.

As an example, let us consider dissipative Linear Complementarity Systems(LCS) [18]:

$$
\left\{\begin{array}{l}
\dot{x}=A x+B \lambda \\
0 \leq y=C x \perp \lambda \geq 0
\end{array}\right.
$$

where $(A, B, C)$ satisfies a) above, $y, \lambda \in \mathbb{R}^{m}$, and $C x(0) \geq 0$. The second line in (6) is a set of complementarity conditions between $y$ and $\lambda$, stating that both these terms have to remain non-negative and orthogonal one to each other. The LCS in (6) can be equivalently rewritten as in (5) with $\varphi(y)=\psi_{\left(\mathbb{R}^{+}\right)^{m}}(y)$.

Remark 2 It is interesting to note a close connection between the system in (5) and Moreau's first order sweeping process [10] [17], i.e. evolution problems of the form $\dot{x}(t) \in$ $-\partial \psi_{C(t)}(x(t))$ for some set $C(t)$. The systems we will deal with in the next section are second order sweeping processes. Incidentally lemma 1 sheds a new light on the result in [18] since it shows that initial jumps in the solution $x(t)$ are not needed if $x(0)$ belongs to the admissible set $\{x \mid C x \in \operatorname{dom} \partial \varphi\}$. The results in [18] allow a feedthrough term in $y$ and provide necessary and sufficient conditions for absence of initial jumps. The present paper doesn't allow a feedthrough term and gives only sufficient conditions for non initial jumps, however more general functions $\varphi(\cdot)$ are considered. Finally lemma 1 is related to the uniqueness result in [9] where the graph $\partial \varphi$ is a relay characteristic and the transfer matrix $G(s)$ is a P-matrix [22] for sufficiently large $s \in \mathbb{R}$. SPR transfer matrices satisfy such requirements, but we considered more general characteristics. 
Finally let us note in passing that lemma 1 applies to nonlinear systems as $\dot{x}=-\sum_{k=0}^{n} x^{2 k+1}-$ $y_{L}, y=x, y_{L} \in \partial \varphi(y), x \in \mathbb{R}$. Indeed the dynamics $-y_{L} \mapsto y$ is strictly dissipative with storage function $\frac{x^{2}}{2}$, so that $P=1$ and $z=x$.

Let us notice that $y \in \operatorname{dom} \partial \varphi$. Finally there exists a Lebesgue integrable function $w(t)$ such that $x(t)=\int w(\tau) d \tau$, where $d \tau$ is the Lebesgue measure. Hence $d x=w(t) d t$ as an equality of measures.

Lemma 2 Let assumptions a), b) hold, the initial data be such that $C x(0) \in \operatorname{dom} \partial \varphi$, and assume that the graph of $\partial \varphi$ contains $(0,0)$. Then: i) $x=0$ is the unique solution of the generalized equation $A x \in B \partial \varphi(C x)$ ii) The fixed point $x=0$ of the system in (4) is exponentially stable.

Proof: The proof of part i) is as follows. First of all notice that $x=0$ is indeed a fixed point of the dynamics with no control, since $0 \in B \partial \varphi(0)$. Now $A x \in B \partial \varphi(C x) \Rightarrow$ $P A x \in P B \partial \varphi(C x) \Rightarrow x^{T} P A x=x^{T} \partial g(x)$, where $g(x)=\varphi(C x)$ [6, p.225], $g(\cdot)$ is convex [6, p.38], and we used assumption a). The multivalued mapping $\partial g(x)$ is monotone since $g(\cdot)$ is convex. Thus $x^{T} \partial g(x) \geq 0$ for all $x \in \mathbb{R}^{n}$. Now there exists $Q=Q^{T}>0$ such that $x^{T} P A x=-\frac{1}{2} x^{T} Q x<0$ for all $x \neq 0$. Clearly then $x$ satisfies the generalized equation only if $x=0$.

Let us now prove part ii). Consider the candidate Lyapunov function $W(x)=\frac{1}{2} x^{T} P x$. From lemma 1 it follows that the dynamics in (4) possesses on $[0,+\infty)$ a solution $x(t)$ which is $\mathrm{AC}$, and whose derivative $\dot{x}(t)$ exists a.e.. The same applies to $W$ which is $\mathrm{AC}[23, \mathrm{p} .189]$. Differentiating along the closed-loop trajectories we get

$$
\begin{aligned}
\frac{d W}{d t}(x(t)) & \stackrel{\text { a.e. }}{=} x^{T} P w \\
& =x^{T} P\left(A x-B y_{L}\right)=-x^{T} Q x-x^{T} P B y_{L} \\
& =-x^{T} Q x-x^{T} C^{T} y_{L}
\end{aligned}
$$

where $y_{L}$ is any vector that belongs to $\partial \varphi(C x)$. The equality in the first line means that the density of the measure $d(W \circ x)$ with respect to the Lebesgue measure $d t$ (which exists since $W(x(t))$ is $\mathrm{AC}$ ) is the function $x^{T} P w$. Consequently $\frac{d W}{d t}+x^{T} Q x \in-x^{T} C^{T} \partial \varphi(C x)=$ $-x^{T} \partial g(x)$ a.e.. Let us consider any $z \in \partial g(x)$. One gets $\frac{d W}{d t} \stackrel{\text { a.e. }}{=}-x^{T} Q x-x^{T} z \leq-x^{T} Q x$ from the property of monotone multivalued mappings and since $(x, z)=(0,0)$ belongs to the graph of $\partial g(x)$. The set of time instants at which the inequality $\frac{d W}{d t} \leq-x^{T} Q x$ is not satisfied is negligible in the Lebesgue measure. It follows that the function of time $W(\cdot)$, which is continuous, is nonincreasing. Thus one has $W(t)-W(0)=\int_{0}^{t}\left(-x^{T} Q x-\right.$ $\left.x^{T} z\right) d \tau \leq-\int_{0}^{t} x^{T} Q x d \tau$. Consequently $\frac{1}{2} \lambda_{\min }(P) x^{T} x \leq W(0)-\int_{0}^{t} \lambda_{\min }(Q) x^{T} x d \tau$, where $\lambda_{\min }(\cdot)$ is the smallest eigenvalue. By the Gronwall's lemma one gets that $\frac{1}{2} \lambda_{\min }(P) x^{T} x \leq$ $W(0) \exp \left(-2 \frac{\lambda_{\min }(Q)}{\lambda_{\min }(P)} t\right)$ which concludes the proof. 
Remark 3 Related worsk can be found in [28] and in [30, theorem 10.5.31], which appears as a particular case of lemma 2. Other extensions for the passivity theorem with feedback branches that may contain various operators and nonlinearities, can be found in [21]. Some of the operators $y \mapsto y_{L}$ considered in [21] encompass the ones considered in this paper (like the saturation in figure $3(\mathrm{~b})$ ). However multivalued mappings are not included in the framework of [21], and neither are graphs including unilateral constraints as in figure 3 (c) (e) (f).

\section{The Lagrange-Dirichlet theorem}

Let us consider the following class of unilaterally constrained mechanical systems

$$
\left\{\begin{array}{l}
M(q) \ddot{q}+F(q, \dot{q})=\nabla h(q) \lambda \\
0 \leq h(q) \perp \lambda \geq 0 \\
\dot{q}\left(t_{k}^{+}\right)=-e \dot{q}\left(t_{k}^{-}\right)+(1+e) \operatorname{prox}_{M\left(q\left(t_{k}\right)\right)}\left[\dot{q}\left(t_{k}^{-}\right), V\left(q\left(t_{k}\right)\right)\right]
\end{array}\right.
$$

In (8) $M(q)=M^{T}(q)>0$ is the $n \times n$ inertia matrix, $F(q, \dot{q})=C(q, \dot{q}) \dot{q}+\frac{\partial U}{\partial q}(q)$ where $C(q, \dot{q}) \dot{q}$ denotes centripetal and Coriolis generalized forces, whereas $U(q)$ is a smooth potential energy from which conservative forces derive. The impact times are generically denoted as $t_{k}$, the left-limit $\dot{q}\left(t_{k}^{-}\right) \in-V\left(q\left(t_{k}\right)\right)$ whereas the right-limit $\dot{q}\left(t_{k}^{+}\right) \in V\left(q\left(t_{k}\right)\right)$. The third line in (8) is a collision mapping that relates pre- and post-impact generalized velocities, and $e \in[0,1]$ is a restitution coefficient [13]. The notation $\operatorname{prox}_{M(q)}$ means the proximation in the kinetic metric, i.e. the metric defined as $x^{T} M(q) y$ for $x, y \in \mathbb{R}^{n}$ : the vector $\frac{\dot{q}\left(t_{k}^{+}\right)+e \dot{q}\left(t_{k}^{-}\right)}{1+e}$ is the closest vector to the pre-impact velocity, inside $V\left(q\left(t_{k}\right)\right)$ (it can therefore be computed through a quadratic programme) [8]. In particular the impact law in (8) implies that the kinetic energy loss at time $t_{k}$ satisfies (see [13], [4, p.199, p.489] [12])

$$
T_{L}\left(t_{k}\right)=-\frac{1}{2} \frac{1-e}{1+e}\left(\dot{q}\left(t_{k}^{+}\right)-\dot{q}\left(t_{k}^{-}\right)\right)^{T} M\left(q\left(t_{k}\right)\right)\left(\dot{q}\left(t_{k}^{+}\right)-\dot{q}\left(t_{k}^{-}\right)\right) \leq 0
$$

Remark 4 The formulation of the unilateral constraints in (8) does not encompass all closed domains $\Phi=\{q \mid h(q) \geq 0\}$, as simple non-convex cases with so-called reentrant corners prove [11]. It can be used to describe admissible domains $\Phi$ which are defined either by a single constraint (i.e. $m=1$ ), or with $m<+\infty$ where convexity holds at nondifferentiable points of the boundary $\partial \Phi$ (such sets are called regular [15]). It is easy to imagine physical examples that do not fit within this framework, e.g. a ladder. We prefer not to analyze this case in this note since neither the modelling nor the mathematical parts are yet fixed for non-differentiable non-convex boundaries $\partial \Phi[25, \mathrm{p} .156][24, \S 9.5]$.

Let us note that the tangent cone $V(q(t))$ is assumed to have its origin at $q(t))$ so that $0 \in V(q(t))$ to allow for post-impact velocities tangential to the admissible set boundary $\partial \Phi$. 
The second line in (8) is a set of complementarity conditions between $h(q)$ and $\lambda$, stating that both these terms have to remain non-negative and orthogonal one to each other. We assume that $h(\cdot): \mathbb{R}^{n} \mapsto \mathbb{R}^{m}$ is differentiable, the gradient $\nabla h(q)$ is not zero at the contact configurations $h(q)=0$, and the vectors $\nabla h_{i}, 1 \leq i \leq m$, are independent. We further assume that the functions $h(\cdot), F(q, \dot{q})$, and $M(q)$ are piecewise real analytic. Then the following results hold:

i) Solutions of (8) exist on $[0,+\infty)$ such that $q(\cdot)$ is absolutely continuous (AC), whereas $\dot{q}(\cdot)$ is right-continuous of local bounded variation (RCLBV). In particular the left and right-limits of these functions exist everywhere [12].

ii) The function $q(\cdot)$ cannot be supposed to be everywhere differentiable. One has $q(t)=$ $q(0)+\int_{0}^{t} v(s) d s$ for some function $v(\cdot) \stackrel{\text { a.e. }}{=} \dot{q}(\cdot)$. Moreover $\dot{q}\left(t^{+}\right)=v\left(t^{+}\right)$and $\dot{q}\left(t^{-}\right)=$ $v\left(t^{-}\right)[10]$.

iii) Solutions are unique [12] (however in general they do not depend continuously on the initial conditions).

iv) The acceleration $\ddot{q}$ is a measure $d v$, which is the sum of two measures [12]: an atomic measure $d \mu_{a}$, and a Lebesgue integrable function which we denote $\ddot{q}(\cdot)$, i.e. $d v=$ $d \mu_{a}+\ddot{q}(t) d t$. The atoms correspond to the impact times [8]. See remark 5 for some comments on this decomposition.

v) The set of impact times is countable. In many applications one has $d \mu_{a}=\sum_{k \geq 0}\left[\dot{q}\left(t_{k}^{+}\right)-\right.$ $\left.\dot{q}\left(t_{k}^{-}\right)\right] \delta_{t_{k}}$, where $\delta_{t}$ is the Dirac measure and the sequence $\left\{t_{k}\right\}_{k \geq 0}$ can be ordered, i.e. $t_{k+1}>t_{k}$. However phenomena like accumulations of left-accumulations of impacts may exist (at least bounded variation does not preclude them). In any case the ordering may not be possible. This is a sort of complex Zeno behaviour $\left({ }^{3}\right)$. In the case of elastic impacts $(e=1)$ it follows from [12, Prop.4.11] that $t_{k+1}-t_{k} \geq \delta>0$ for some $\delta>0$. Hence solutions are piecewise continuous in this case.

vi) Any quadratic function $W(\cdot)$ of $\dot{q}$ is itself RCLBV, hence its derivative is a measure $d W$ [8]. Consequently $d W \leq 0$ has a meaning and implies that the function $W(\cdot)$ does not increase [26, p.101].

These results enable one to lead a stability analysis safely. Let us now introduce a new formulation of the dynamics in (8), which can be written as the following Measure Differential Inclusion (MDI) [8]

$$
-M(q(t)) d v-F\left(q(t), v\left(t^{+}\right)\right) d t \in \partial \psi_{V(q(t))}(w(t)) \subseteq \partial \psi_{\Phi}(q(t))
$$

where $w(t)=\frac{v\left(t^{+}\right)+e v\left(t^{-}\right)}{1+e} \in \partial V(q(t))$ from (8). If $e=0$ then $w(t)=v\left(t^{+}\right)$, if $e=1$ then $w(t)=\frac{v\left(t^{+}\right)+v\left(t^{-}\right)}{2}$. Moreover when $v(\cdot)$ is continuous then $w(t)=v(t)$. The term MDI

\footnotetext{
${ }^{3}$ I.e. all phenomena involving an infinity of events in a finite time interval, and which occur in various types of hybrid systems like Filippov's inclusions, etc [14].
} 
has been coined by Moreau, and (10) may also be called Moreau's second order sweeping process [10]. The inclusion in the right-hand-side of (10) is proved in appendix A. When $\dot{q}(t)$ is discontinuous, (10) implies that Moreau's collision rule in (8) is satisfied. This can be proved using basic tools from convex analysis, see appendix B. The term $\psi_{V(q(t))}(w(t))$ can be interpreted as a velocity potential and its subdifferential $\partial \psi_{V(q(t))}(w(t))$ is depicted in figure 4 in special cases. The MDI in (10), whose left-hand-side is a measure and whose righthand-side is a cone, has the following meaning [17] [13]: there exists a positive measure $d \mu$ such that both $d t$ and $d v$ possess densities with respect to $d \mu$, denoted respectively as $\frac{d t}{d \mu}(\cdot)$ and $\frac{d v}{d \mu}(\cdot)$. One also has $\frac{d t}{d \mu}(t)=\lim _{\epsilon \rightarrow 0, \epsilon>0} \frac{d t([t, t+\epsilon])}{d \mu(t t, t+\epsilon])}[29][17$, p.9], which shows the link with the usual notion of a derivative. The choice of $d \mu$ is not unique because the right-hand-side is a cone [8]. However by the Lebesgue-Radon-Nikodym theorem [23], the densities $\frac{d t}{d \mu}(\cdot)$ and $\frac{d v}{d \mu}(\cdot)$ are unique functions for a given $d \mu$. To shed some light on this, let us consider for instance $d \mu=d t+\Sigma_{k \geq 0} \delta_{t_{k}}$, which corresponds to applications where the system is subject to impacts at times $t_{k}$ and otherwise evolves freely. Then $\frac{d t}{d \mu}\left(t_{k}\right)=0$ (the Lebesgue measure $d t$ and the Dirac measure $\delta_{t}$ are mutually singular) whereas $\frac{d v}{d \mu}\left(t_{k}\right)=v\left(t_{k}^{+}\right)-v\left(t_{k}^{-}\right)\left(t_{k}\right.$ is an atom of the measure $d v)$. When $t \neq t_{k}$ then $\frac{d t}{d \mu}(t)=1$ and $\frac{d v}{d \mu}(t)=\dot{v}(t)$.

Therefore the meaning of (10) is that there exists a positive measure $d \mu$ with respect to which both $d t$ and $d v$ possess densities, and

$$
-M(q(t)) \frac{d v}{d \mu}(t)-F\left(q(t), v\left(t^{+}\right)\right) \frac{d t}{d \mu}(t) \in \partial \psi_{V(q(t))}(w(t)) \subseteq \partial \psi_{\Phi}(q(t))
$$

holds $d \mu$-almost everywhere. In a sense, densities replace derivatives, for measures. When dealing with measure differential equations or inclusions, it is then natural to manipulate densities instead of derivatives. In general one can choose $d \mu=|d v|+d t[17, \mathrm{p} .90]$, where $|d v|$ is the absolute value of $d v$, or $d \mu=\|v(t)\| d t+d \mu_{a}$, or $d \mu=d t+d \mu_{a}$. It is fundamental to recall at this stage, that the solution of (11) does not depend on this choice. For instance, if $d \mu=\|v(t)\| d t+d \mu_{a}$ then for all $t \neq t_{k}, \frac{d t}{d \mu}(t)=\frac{1}{\|v(t)\|}$ and $\frac{d v}{d \mu}(t)=\frac{\ddot{q}(t)}{\|v(t)\|}$. Whereas if $d \mu=d t+d \mu_{a}$ then for all $t \neq t_{k}, \frac{d t}{d \mu}(t)=1$ and $\frac{d v}{d \mu}(t)=\ddot{q}(t)$.

Remark 5 The above mathematical framework is more than just a mathematical fuss. Indeed as noted in [8], introducing the velocity into the right-hand-side of the dynamics as done in (10), not only allows one to get a compact formulation of the nonsmooth dynamics (see figure 6 in this respect), but it also paves the way towards the consideration of friction in the model. In turn it is clear that introducing friction, is likely to complicate the dynamics. Especially the above framework paves the way towards more complex cases where the measure $d v$ may contain a third term $d \mu_{n a}$ which is a nonatomic measure singular with respect to the Lebesgue measure $d t$. In summary the dynamics in (11) is rich enough to encompass complex behaviours involving solutions which may be far from merely piecewise continuous as for the models and stability analysis in [3]. This is a consequence of replacing functions by the more general notion of measure, at the price of a more involved model. In 
fact using measures allows one to encompass somewhat complex Zeno behaviours occurring in unilaterally constrained mechanical systems in a rigorous manner.

In the case of unconstrained Lagrangian mechanical systems, the Lagrange-Dirichlet theorem states that the equilibrium point $\left(q_{0}, 0\right)$ is locally stable if the potential energy $U(q)$ has a strict minimum at $q_{0}$. First notice that since $F(q, 0)=\frac{\partial U}{\partial q}$, fixed points of (10) satisfy the generalized equation $0 \in \partial \psi_{\Phi}\left(q_{0}\right)+\frac{\partial U}{\partial q}\left(q_{0}\right)$ which in particular implies $q_{0} \in \Phi$. Conditions under which such a generalized equation possess at least one solution, and numerical algorithms to compute one solution, exist [27]. In the following we shall assume for simplicity that the solutions are isolated.

Lemma 3 Consider a mechanical system as in (8). Then if $\psi_{\Phi}(q)+U(q)$ has a strict minimum at $q_{0}$, the equilibrium point $\left(q_{0}, 0\right)$ is Lyapunov stable.

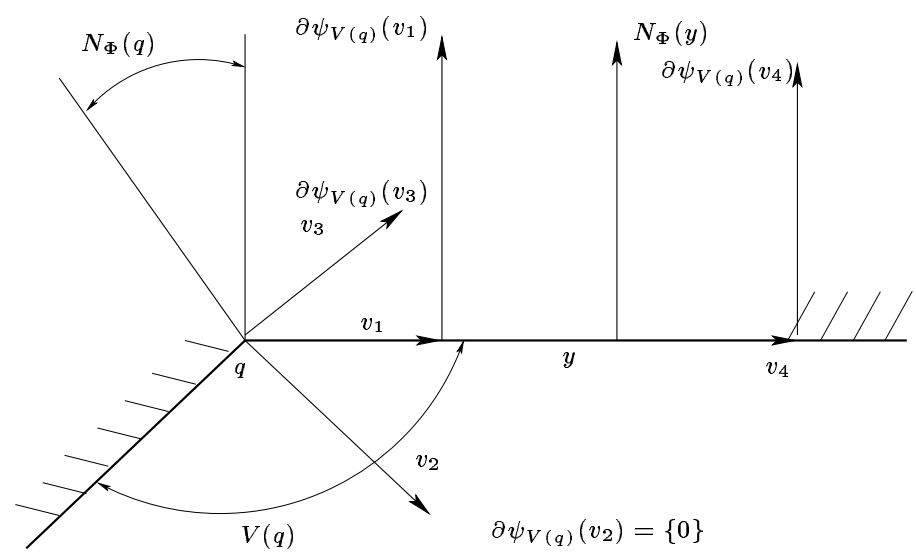

$\Phi$

Figure 4: Moreau's velocity potential.

Let us note that $\Phi$ needs not be convex in general (for instance the equilibrium may exist in $\operatorname{Int}(\Phi)$, or it may belong to $\partial \Phi$ but be forced by the continuous dynamics, see figure 5 for planar examples with both convex and non-convex $\Phi$; it is obvious that in the depicted non-convex case all points $\left(q_{0}, 0\right)$ with $q_{0} \in \partial \Phi$ are fixed points of the dynamics).

Proof: The proof may be led as follows. Let us consider the nonsmooth Lyapunov candidate function

$$
W(q(t), \dot{q}(t))=\frac{1}{2} \dot{q}(t)^{T} M(q(t)) \dot{q}(t)+\psi_{\Phi}(q(t))+U(q(t))-U\left(q_{0}\right)
$$

INRIA 

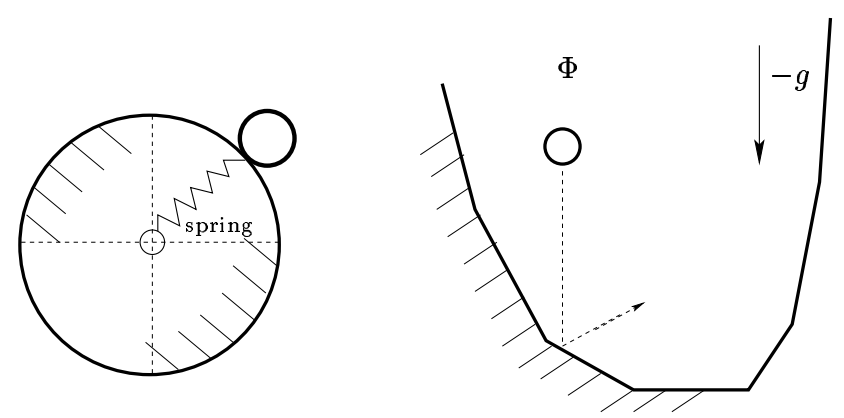

$\Phi$

Figure 5: Convex and nonconvex admissible sets.

Since the potential $\psi_{\Phi}(q)+U(q)$ has a strict minimum at $q_{0}$ equal to $U\left(q_{0}\right)$, this $W$ is positive definite on the whole state space. Moreover since (10) secures that $q(t) \in \Phi$ for all $t \geq 0$, it follows that $\psi_{\Phi}(q(t))=0$ for all $t \geq 0$, and $W(q(t), \dot{q}(t)) \leq \beta(\|q(t)\|,\|\dot{q}(t)\|)$ for some class-K function $\beta(\cdot)$, on $\Phi(\ni q(t)$ for all $t \geq 0)$. However its derivative on the boundary $\partial \Phi$ is not zero, see (2). The potential function $\psi_{\Phi}(q(t))+U(q(t))$ is continuous since $q(\cdot)$ is continuous and $q(\cdot) \in \Phi$. Let us examine the variation of $W(q, \dot{q})$ along trajectories of (11). In view of the above discussion, one can characterize the measure $d W$ by its density with respect to $d \mu$ and the function $W$ decreases if its density $\frac{d W}{d \mu}(t) \leq 0$ for all $t \geq 0$. We recall Moreau's rule for differentiation of quadratic functions of RCLVB functions [17, p.8-9]: let $u(\cdot)$ be RCLBV, then $d\left(u^{2}\right)=\left(u^{+}+u^{-}\right) d u$ where $u^{+}$and $u^{-}$are the right-limit and left-limit functions of $u(\cdot)$. Let us now compute the density of the measure $d W$ with respect to $d \mu$ :

$$
\begin{aligned}
\frac{d W}{d \mu}(t) & =\frac{1}{2}\left[\dot{q}\left(t^{+}\right)+\dot{q}\left(t^{-}\right)\right]^{T} M(q(t)) \frac{d v}{d \mu}(t)+\dot{q}\left(t^{+}\right)^{T} z_{2} \frac{d t}{d \mu}(t)+\frac{\partial U}{\partial q} \frac{d q}{d \mu}(t) \\
& +\frac{1}{2} \frac{\partial}{\partial q}\left(\dot{q}\left(t^{+}\right)^{T} M(q(t)) \dot{q}\left(t^{+}\right)\right) \frac{d q}{d \mu}(t)
\end{aligned}
$$

where $d q=v(t) d t$ since the function $v(\cdot)$ is Lebesgue integrable, and $z_{2} \in \partial \psi_{\Phi}(q(t))$.

Let us now choose $d \mu=d t+d \mu_{a}$. Since $\frac{d t}{d \mu}\left(t_{k}\right)=0$ and $\frac{d q}{d \mu}\left(t_{k}\right)=0$ whereas $\frac{d v}{d \mu}\left(t_{k}\right)=$ $\beta\left[v\left(t_{k}^{+}\right)-v\left(t_{k}^{-}\right)\right]=\beta\left[\dot{q}\left(t_{k}^{+}\right)-\dot{q}\left(t_{k}^{-}\right)\right]$for some $\beta>0$, it follows from (13) that at impact times one gets

$$
\frac{d W}{d \mu}\left(t_{k}\right)=\frac{1}{2} \beta\left[\dot{q}\left(t_{k}^{+}\right)+\dot{q}\left(t_{k}^{-}\right)\right]^{T} M(q(t))\left[\dot{q}\left(t_{k}^{+}\right)-\dot{q}\left(t_{k}^{-}\right)\right]=\beta T_{L}\left(t_{k}\right) \leq 0
$$

where $T_{L}\left(t_{k}\right)$ is in (9). Let $\dot{M}(q, \dot{q})=\frac{d}{d t}[M(q(t))]$. Let us use the expression of $F(q, \dot{q})$ given after (8), and let us assume that Christoffel's symbols of the first kind are used to 
compute the vector $C(q, \dot{q}) \dot{q}$. Then $C(q, \dot{q}) \dot{q}=\dot{M}(q, \dot{q})-\frac{1}{2}\left[\frac{\partial}{\partial q}\left(\dot{q}^{T} M(q(t)) \dot{q}\right)\right]^{T}$ and the matrix $\dot{M}(q, \dot{q})-2 C(q, \dot{q})$ is skew-symmetric [31, §1.2]. Now if $t \neq t_{k}$, one gets $\frac{d v}{d \mu}(t)=$ $\dot{v}(t)=\ddot{q}(t)$ and $\frac{d t}{d \mu}(t)=1[17, \mathrm{p} .76]$ and one can calculate from (13), using the dynamics and the skew-symmetry property:

$$
\begin{aligned}
\frac{d W}{d \mu}=\frac{d W}{d t} & =-\dot{q}^{T} C(q, \dot{q}) \dot{q}+\frac{1}{2} \dot{q}^{T} \dot{M}(q, \dot{q}) \dot{q}-\dot{q}^{T} z_{1}+\dot{q}^{T} z_{2} \\
& =-\dot{q}^{T} z_{1}+\dot{q}^{T} z_{2}
\end{aligned}
$$

where $z_{1} \in \partial \psi_{V(q(t))}(w(t))$ and $W(\cdot)$ is defined in (12). To simplify the notation we have dropped arguments in (15), however $\dot{q}$ is to be understood as $\dot{q}(t)=\dot{q}\left(t^{+}\right)$since $t \neq t_{k}$. Now since for all $t \geq 0 \dot{q}\left(t^{+}\right) \in V(q)$ [8] which is polar to $\partial \psi_{\Phi}(q(t))$ one gets $z_{2}^{T} \dot{q}\left(t^{+}\right) \leq 0$, and from the inclusion in appendix A it follows that $z_{1}^{T} \dot{q}\left(t^{+}\right) \leq 0$ as well. Therefore the measure $d W$ is non-positive. Consequently the function $W(\cdot)$ is non-increasing [26, p.101] and lemma 3 is proved.

Remark 6 - The inclusion of the indicator function $\psi_{\Phi}(q(t))$ in the Lyapunov function not only guarantees its positive definiteness (which anyway is assured along solutions of (11) which remain in $\Phi$ ), but it also allows one to consider cases where the smooth potential has a minimum that is outside $\Phi$. Saying " $\psi_{\Phi}(q)+U(q)$ has a strict minimum at $q_{0}$ " is the same as saying " $U(q)$ has a strict minimum at $q_{0}$ inside $\Phi$ ". Since the indicator function has originally been introduced by Moreau as a potential associated to unilateral constraints, it finds here its natural use.

- As alluded to above, when $e=1$ in (8), the sequence of impact times $\left\{t_{k}\right\}_{k \geq 0}$ can be ordered, $d \mu_{a}=\sum_{k>0} \delta_{t_{k}}$, and velocities are piecewise continuous. Then a much simpler formulation can be adopted by separating continuous motion phases occurring on intervals $\left(t_{k}, t_{k+1}\right)$ from impact times. The stability framework in [3] is sufficient in such a case. The system is therefore non-Zeno for $e=1$ and with the above assumptions.

- One doesn't need to make further assumptions on the measure $d \mu_{a}$ to conclude, and one sees that this conclusion is obtained directly applying general differentiation rules of RCLBV functions. The dynamics might even contain dense sets of velocity discontinuities, (13) and (14) would continue to hold. This shows that using the MDI formalism in (10) or (11) places the stability analysis in a much more general perspective than, say, restricting $\dot{q}(\cdot)$ to be piecewise continuous.

Let us note that the dynamics in (10) has the interpretation as in figure 6, where $\xi \in \partial \psi_{V(q(t))}(w(t))$. Since $\partial \psi_{V(q(t))}(w(t)) \subseteq N_{\Phi}(q)=V^{\star}(q)$ (the cone polar to $V(q)[6]$ ), the feedback loop in figure 6 contains the cone complementarity problem [22]

$$
N_{\Phi}(q) \supseteq \partial \psi_{V(q(t))}(w(t)) \ni \xi \perp w(t) \in V(q)
$$


When $m=1$ and $q \in \partial \Phi$, one has $V(q)=\mathbb{R}^{+}$and $N_{\Phi}(q)=\mathbb{R}^{-}$in a suitable frame attached to $q$, and the graph of the multivalued mapping is the so-called corner law. In general this is an example of an $m$-dimensional monotone multivalued mapping $w(t) \mapsto \xi$. Thus lemma 3 extends the absolute stability problem studied in the foregoing section towards another type of dynamical systems. It is noteworthy that the feedback loop in figure 6 contains both the complementarity conditions and the collision mapping in (8). A quite similar structure can be found for the dynamics in (6) with the state re-initialization mapping proposed in [18], which is an extension of (16).

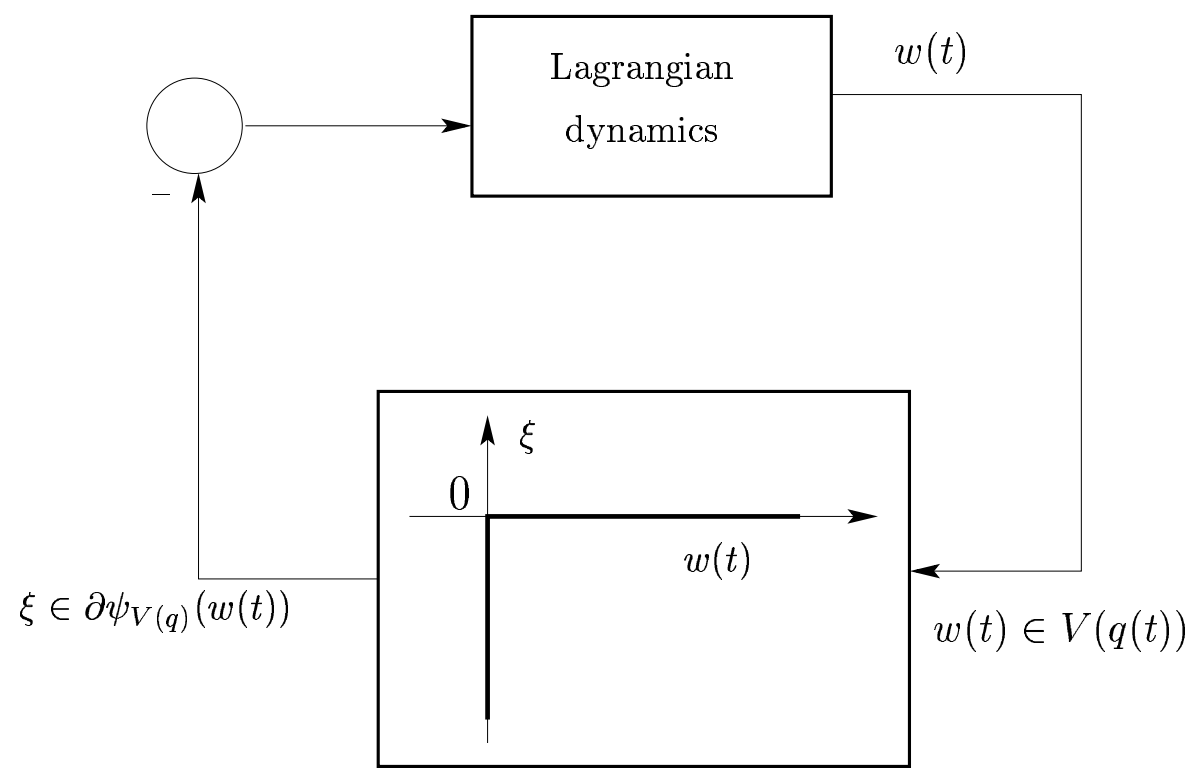

Figure 6: Unilaterally constrained Lagrangian system.

The Lagrange-Dirichlet theorem serves as a basis for the stabilization of unconstrained mechanical systems, both in the regulation and the tracking cases [2]. In the regulation case, one may shape the potential energy so that the new equilibrium of the closed-loop system is the desired one. Lemma 3 can be used to that aim by shaping the potential $\psi_{\Phi}(q)+U(q)$ to a new closed-loop potential $\psi_{\Phi}(q)+\tilde{U}(\tilde{q})$ via a PD control where $\tilde{q}=q-q_{d}$ and $q_{d}$ is some constant desired position. However the closed-loop fixed points have to satisfy the generalized equation $0 \in \partial \psi_{\Phi}\left(q_{0}\right)+\frac{\partial \tilde{U}}{\partial \tilde{q}}\left(\tilde{q}_{0}\right)$ with $\tilde{q}_{0}=q_{0}-q_{d}$, which reflects the fact that $q_{0} \in \Phi$. Extension towards tracking is more complex but can be done [4] [5]. 
Example 1 Let us consider the system in figure 7 whose dynamics is given by

$$
\left\{\begin{array}{l}
m \ddot{x}=-f \dot{x}-c x \\
m \ddot{y}=-m g+\lambda, \quad 0 \leq y \perp \lambda \geq 0 \\
\dot{y}\left(t_{k}^{+}\right)=-e \dot{y}\left(t_{k}^{-}\right) \text {when } y\left(t_{k}\right)=0
\end{array}\right.
$$

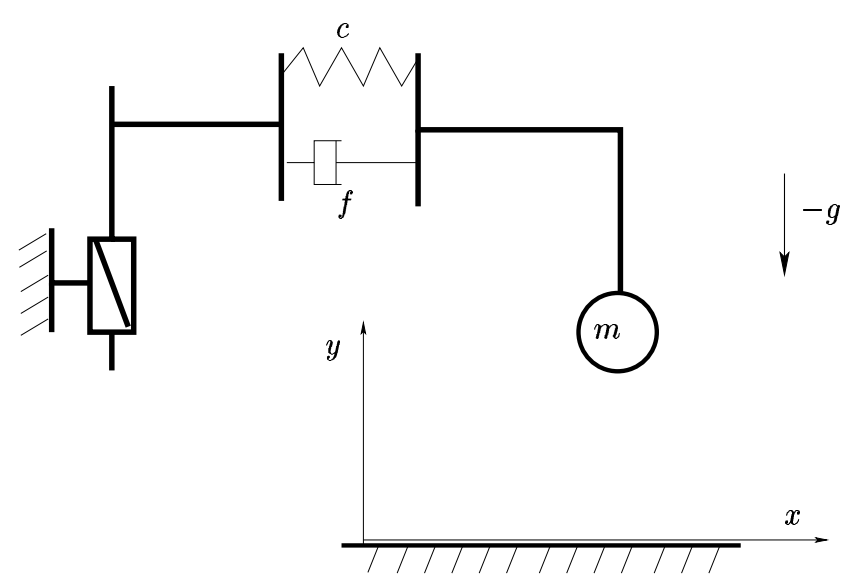

Figure 7: A simple 2-dof system.

Obviously $\left(q, \dot{q}\left(t^{+}\right)\right)=(0,0)$ is the unique fixed point of $(17)$ and one can check that the same dynamics with $y \leq 0$ no longer possesses any fixed point. For any $y(0)>0$ and $e \in(0,1)$ the sequence of impact times has a finite accumulation $t_{\infty}$ [4]. The function in (12) is equal to

$$
W(q, \dot{q})=\frac{1}{2} m \dot{x}^{2}+\frac{1}{2} m \dot{y}^{2}+\psi_{\mathbb{R}^{+}}(y)+m g y+\frac{1}{2} c x^{2}
$$

One may check that this function has the properties required in the proof of lemma 3.

\section{Conclusions}

This note examines the extension of the absolute stability problem and of the LagrangeDirichlet theorem, to the case when nonlinearities in the feedback loop are multivalued monotone mappings. Some of the considered systems may also be recast in the framework of complementarity systems, which in turn may be seen as a special class of hybrid dynamical systems. Another, fruitful point of view, is that of differential inclusions, with or without 
measures. This class of systems encompasses mechanical systems with unilateral constraints and impacts, as well as dissipative linear complementarity systems without feedthrough term in the output equation. The well-posedness is carefully examined before studying the stability. Roughly speaking, the first class of nonsmooth systems studied in this paper correspond to relative degree one systems, while the second class are relative degree two systems. In particular a new existence and uniqueness proof for a class of nonsmooth dissipative systems, is given. Further extensions of the results towards more complex mechanical systems with set valued force laws [24] [25], seem quite interesting.

Acknowledgements: I would like to thank Manuel Monteiro Marques (CMAF Lisbon) for his help in the proof in appendix A, and Claude Lemaréchal (INRIA) for his help in the proof of lemma 1 .

\section{A Proof of Moreau's inclusion}

For convenience let us rewrite the following definitions for a convex set $\Phi$ :

$$
N_{\Phi}(q)=\left\{z \mid z^{T} \xi \leq 0, \forall \xi \in V(q)\right\}
$$

which is an alternative definition to the one given in section 2 , and

$$
\partial \psi_{V(q)}(w)=\left\{z \mid z^{T}(\eta-\dot{q}) \leq 0, \forall \eta \in V(q)\right\}
$$

Since $V(q)$ as it is defined in (3) (replace $K$ by $\Phi$ ) is a cone and since $\dot{q} \in V(q)$, one can choose $\eta=\xi+\dot{q}$ with $\xi \in V(q)$ as a particular value for $\eta$. Thus if $z \in \partial \psi_{V(q)}(w)$ one gets $z^{T} \eta \leq z^{T} \dot{q}\left(t^{+}\right)$and introducing $\eta=\xi+\dot{q}$, one gets $z^{T} \xi \leq 0$ so that $z \in N_{\Phi}(q)$. Therefore Moreau's inclusion in (10) is proved.

Let us note [10] that the cones are to be understood as being attached to the same origin in the inclusion (for instance in figure 4, one has $\partial \psi_{V(q)}\left(v_{1}\right) \subset N_{\Phi}(q)$ ). Moreover some natural identifications between spaces (the dual $T_{\dot{q}}^{*} T_{q} \mathcal{Q}$ at $\dot{q}$ of the tangent space $T_{q} \mathcal{Q}$ at $q$ to the configuration space $\mathcal{Q}$, and the cotangent space $T_{q}^{*} \mathcal{Q}$ ) have been made, thanks to the linear structure of these spaces in which the cones $\partial \psi_{V(q)}(\cdot)$ and $N_{\Phi}(q)$ are defined. This allows to give a meaning to the inclusion in (10). This is just a generalization of the wellknown identification between the space of velocities and that of forces acting on a particle in a 3-dimensional space, which both are identified with $\mathbb{R}^{3}$. More details are in [12] and [8].

\section{B The MDI at impact times}

Let $x$ and $z$ be two vectors of a linear Euclidean space $E, V$ be a closed convex cone of $E$, and $N$ be the polar cone to $V$. Then from Moreau's lemma of the two cones [6, p.340] [4, lemma D1], one has $(x-z) \in \partial \psi_{V}(x) \Longleftrightarrow x=\operatorname{prox}[V, z] \Longleftrightarrow z-x=\operatorname{prox}[N, z]$. 
Times $t_{k}$ are atoms of the measure $d v$ in (10). Via a suitable base change, the kinetic metric at an impact time can be considered as a Euclidean metric since $q(\cdot)$ is continuous at $t_{k}$, and in particular all the identifications between various dual spaces can be done. One gets from (10): $-M\left(q\left(t_{k}\right)\right)\left[\dot{q}\left(t_{k}^{+}\right)-\dot{q}\left(t_{k}^{-}\right)\right] \in \partial \psi_{V\left(q\left(t_{k}\right)\right)}\left(w\left(t_{k}^{+}\right)\right) \Longleftrightarrow \dot{q}\left(t_{k}^{+}\right)+e \dot{q}\left(t_{k}^{-}\right)=$ $\operatorname{prox}_{M\left(q\left(t_{k}\right)\right)}\left[V\left(q\left(t_{k}\right)\right),(1+e) \dot{q}\left(t_{k}^{-}\right)\right] \Longleftrightarrow \dot{q}\left(t_{k}^{+}\right)+e \dot{q}\left(t_{k}^{-}\right)=(1+e) \operatorname{prox}_{M\left(q\left(t_{k}\right)\right)}\left[V\left(q\left(t_{k}\right)\right), \dot{q}\left(t_{k}^{-}\right)\right.$, where the second equivalence is proved in [8].

\section{References}

[1] M. Vidyasagar, 1993 Nonlinear Systems Analysis, second edition, Prentice-Hall.

[2] R. Lozano, B. Brogliato, O. Egeland, B. Maschke, 2000 Dissipative Systems Analysis and Control, Springer, Communications and Control Engineering Series, London.

[3] D.D. Bainov, P.S. Simeonov, 1989 Systems with Impulse Effects; Stability, Theory and Applications, Ellis Horwood series in Mathematics and its applications, Wiley.

[4] B. Brogliato, 1999 Nonsmooth Mechanics, Springer Communications and Control Engineering Series, second edition, London. Erratum and addenda available at http://www.inrialpes.fr/bip/people/brogliato/brogli.html

[5] B. Brogliato, S.I. Niculescu, M. Monteiro-Marques, 2000 "On tracking control of a class of complementary-slackness hybrid mechanical systems", Systems and Control Letters, vol.39, no 4, pp.255-266, March.

[6] R.T. Rockafellar, 1970 Convex Analysis, Princeton Landmarks in Mathematics, Princeton University Press, New Jersey.

[7] J.J. Moreau, 1966-1967 Fonctionnelles Convexes, Séminaire sur les équations aux dérivées partielles, Collège de France, Paris.

[8] J.J. Moreau, 1988 "Unilateral contact and dry friction in finite freedom dynamics", in Nonsmooth Mechanics and Applications, CISM Courses and Lectures no 302, J.J. Moreau, P.D. Panagiotopoulos (Eds.), International Centre for Mechanical Sciences, Springer-Verlag., pp.1-82.

[9] Y.J. Lootsma, A.J. van der Schaft, M.K. Camlibel, 1999 "Uniqueness of solutions of linear relay systems", Automatica, vol.35, no 3, pp.467-478.

[10] M. Kunze, M.D.P. Monteiro Marques, 2000 "An introduction to Moreau's sweeping process", in Impacts in Mechanical Systems. Analysis and Modelling, B. Brogliato (Ed.), Springer, Lecture Notes in Physics LNP 551, pp.1-60.

[11] B. Brogliato, 2001 "On the control of nonsmooth complementarity dynamical systems", Phil. Trans. Royal Soc., Mathematical, Physical and Engineering Sciences, special issue on Nonsmooth Mechanics, series A, vol.359, no 1789, pp.2369-2383.

INRIA 
[12] P. Ballard, 2001 "Formulation and well-posedness of the dynamics of rigid-body systems with perfect unilateral constraints", Phil. Trans. Royal Soc., Mathematical, Physical and Engineering Siences, special issue on Nonsmooth Mechanics, series A, vol.359, no 1789, pp.2327-2346.

[13] M. Mabrouk, 1998 "A unified variational model for the dynamics of perfect unilateral constraints", European Journal of Mechanics A/Solids, vol.17, no 5, pp.819-842.

[14] A. van der Schaft, H. Schumacher, 2000 An Introduction to Hybrid Dynamical Systems, Springer, LNCIS 251, London.

[15] F.H. Clarke, 1983 Optimization and Nonsmooth Analysis, Canadian Math. Soc. Series of Monographs and Advanced Texts, Wiley Interscience Publications.

[16] H. Brezis, 1973 Opérateurs Maximaux Monotones, North-Holland, Mathematics Studies.

[17] M.D.P. Monteiro-Marques, 1993 Differential Inclusions in Nonsmooth Mechanical Problems: Shocks and Dry Friction, Birkhauser, Boston, PNLDE 9.

[18] M.K. Camlibel, W.P.M.H. Heemels, J.M. Schumacher, 2002 "On linear passive complementarity systems", European Journal of Control, vol.8, no 3, pp.220-237.

[19] P.D. Panagiotopoulos, 1985 Inequality Problems in Mechanics and Applications. Convex and Nonconvex Energy Functions, Birkhauser Boston.

[20] E. Dimnet, M. Frémond, 2001 "Collisions involving solids and fluids", in Novel Approaches in Civil Engineering, Springer Verlag, M. Frémond, F. Macceri (Eds.).

[21] A. Megretski, A. Rantzer, 1997 "System analysis via integral quadratic constraints", IEEE Transactions on Automatic Control, vol.42, no 6, pp.819-830, June.

[22] R.W. Cottle, J.S. Pang, R.E. Stone, 1992 The Linear Complementarity Problem, Academic Press.

[23] W. Rudin, 1998 Analyse Réelle et Complexe, Dunod, Paris.

[24] Ch. Glocker, 2001 Set-Valued Force Laws, Springer, Lecture Notes in Applied Mechanics vol.1, Berlin Heidelberg.

[25] M. Frémond, 2002 Non-Smooth Thermomechanics, Springer, Berlin Heidelberg.

[26] J. Dieudonné, 1969 Eléments d'Analyse, tome 2, Gauthier-Villars, Paris.

[27] D. Goeleven, G.E. Stavroulakis, G. Salmon, P.D. Panagiotopoulos, 1996 "Solvability theory and projection method for a class of singular variational inequalities: Elastostatic unilateral contact applications", J. of Optimization Theory and Applications, vol.95, no 2. 
[28] D. Goeleven, D. Motreanu, V.V. Motreanu, 2002 "On the stability of stationary solutions of parabolic variational inequalities", to appear in Advances in Nonlinear Variational Inequalities.

[29] J.J. Moreau, M. Valadier, 1986 "A chain rule involving vector functions of bounded variation", J. Funct. Analysis, vol.74, pp.333-345.

[30] A.N. Michel, K. Wang, B. Hu, 2001 Qualitative Theory of Dynamical Systems, second Edition, Marcel Dekker, New-York.

[31] Zodiac, 1996 Theory of Robot Control, Springer-Verlag London, Communications and Control Engineering Series, C. Canudas de Wit, B. Siciliano, G. Bastin (Eds.).

\section{Contents}

1 Introduction $\quad 3$

2 Basic facts in convex and nonsmooth analysis 4

3 The absolute stability problem 6

$\begin{array}{lll}4 & \text { The Lagrange-Dirichlet theorem } & 9\end{array}$

5 Conclusions $\quad 16$

$\begin{array}{ll}\text { A Proof of Moreau's inclusion } & 17\end{array}$

$\begin{array}{ll}\text { B The MDI at impact times } & 17\end{array}$ 


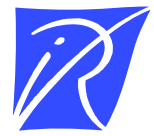

Unité de recherche INRIA Rhône-Alpes

655, avenue de l'Europe - 38330 Montbonnot-St-Martin (France)

Unité de recherche INRIA Futurs : Domaine de Voluceau - Rocquencourt - BP 105 - 78153 Le Chesnay Cedex (France) Unité de recherche INRIA Lorraine : LORIA, Technopôle de Nancy-Brabois - Campus scientifique 615, rue du Jardin Botanique - BP 101 - 54602 Villers-lès-Nancy Cedex (France)

Unité de recherche INRIA Rennes : IRISA, Campus universitaire de Beaulieu - 35042 Rennes Cedex (France) Unité de recherche INRIA Rocquencourt : Domaine de Voluceau - Rocquencourt - BP 105 - 78153 Le Chesnay Cedex (France) Unité de recherche INRIA Sophia Antipolis : 2004, route des Lucioles - BP 93 - 06902 Sophia Antipolis Cedex (France) 